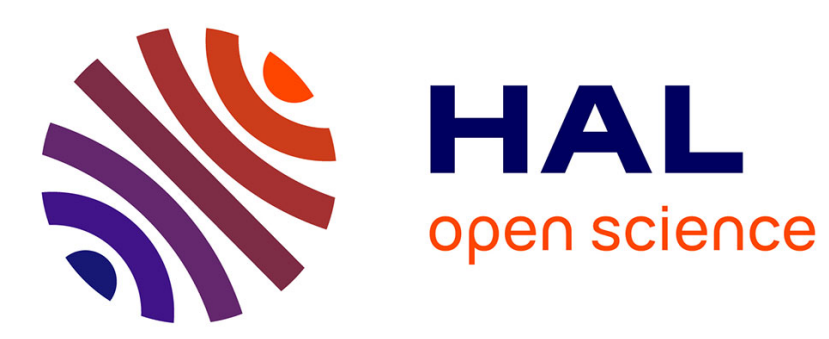

\title{
SC-FDMA with Index Modulation for M2M and IoT Uplink Applications
}

Marwa Chafii, Faouzi Bader, Jacques Palicot

\section{To cite this version:}

Marwa Chafii, Faouzi Bader, Jacques Palicot. SC-FDMA with Index Modulation for M2M and IoT Uplink Applications. 2018 IEEE Wireless Communications and Networking Conference (WCNC), Apr 2018, Barcelona, Spain. 10.1109/wcnc.2018.8377028 . hal-01705717

\section{HAL Id: hal-01705717 https://hal.science/hal-01705717}

Submitted on 9 Feb 2018

HAL is a multi-disciplinary open access archive for the deposit and dissemination of scientific research documents, whether they are published or not. The documents may come from teaching and research institutions in France or abroad, or from public or private research centers.
L'archive ouverte pluridisciplinaire HAL, est destinée au dépôt et à la diffusion de documents scientifiques de niveau recherche, publiés ou non, émanant des établissements d'enseignement et de recherche français ou étrangers, des laboratoires publics ou privés. 


\title{
SC-FDMA with Index Modulation for M2M and IoT Uplink Applications
}

\author{
Marwa Chafii, Carlos Faouzi Bader, and Jacques Palicot \\ IETR/CentraleSupélec, Campus de Rennes, \\ Avenue de la Boulaie, 35510 Cesson-Sévigné, France \\ Email: \{marwa.chafii,faouzi.bader,jacques.palicot\}@centralesupelec.fr
}

\begin{abstract}
One of the most challenging issues of Internet of Things (IoT) devices is energy consumption. A massive deployment of connected widgets is power hungry. Maximizing the energy efficiency of their batteries reduces the maintenance cost and extends their autonomy and then their utility. To address this issue, this paper proposes the introduction of index modulation on single carrier-frequency division multiple access (SC-FDMA) uplink transmissions for cellular IoT and machineto-machine (M2M) networks. It is shown that the proposed SCFDMA with index modulation (SC-FDMA-IM) scheme achieves $50 \%$ of energy efficiency increase, while improving the bit error rate performance by up to $--\mathbf{d B}$ of $E_{b} / N_{0}$ for the same spectral efficiency performance.
\end{abstract}

\section{INTRODUCTION AND MOTIVATIONS}

Machine-to-machine (M2M) communications and more generally internet of things (IoT) communications enable devices to sense their environment, exchange this information among themselves with direct connectivity without human intervention. This data can also be shared actively with humans to allow intelligent decision making. It affects our whole life and our entire ecosystem.

One of the most challenging requirement of countless range of IoT applications, is to achieve energy efficiency high enough to offer ten years of battery lifetime for end devices. Low power wide area (LPWA) [1] technologies have been designed to meet the requirements of M2M and IoT applications in terms of low energy consumption and wide coverage. Unlike traditional cellular technologies, LPWA offers sets of features to low power devices at the expense of low data rates.

The 3rd generation partnership project (3GPP) ${ }^{1}$ enters the M2M and IoT market through the recent proposition of LTE Category M (LTE-M) [2] (also known as enhanced machinetype communications (eMTC)) and Narrow Band-IoT (NBIoT) [3] standards in Release-13. In addition to improving the range, reducing the complexity, and the cost of the devices, these cellular solutions adopt two saving power modes in order to prolong the battery lifetime of the devices. The power saving mode (PSM) and the extended discontinuous (eDRx) reception mode enable end devices to enter in a deep sleep mode for hours or days while maintaining their network registration.

In this paper, we propose index modulation (IM) [4] as a power saving technique for cellular IoT uplink communications. IM technique consists of selecting a set of sub-carriers for each symbol, the selected sub-carriers will carry on the input data symbols while the rest of the sub-carriers is turned

\footnotetext{
${ }^{1}$ More details on http://www.3gpp.org/.
}

off (an idea first reported in [5], [6]). Additional information is coded through the choice of sub-carriers indices in a given symbol. Several variants of indexing have been proposed in the literature in order to increase the spectral efficiency [7], and enhance the bit error rate (BER) performance [8].

Index modulation has been proposed for $5 \mathrm{G}$ wireless networks for the design of downlink transmission protocols [9], [10]. However, we believe that applying IM to the uplink cellular IoT standards is more relevant, since the complexity is offloaded from end devices and kept at the receiver. This application is also motivated by the consideration that IM is more efficient in low modulation orders and for low number of carriers [11].

This paper shows that applying index modulation to SCFDMA for NB-IoT and LTE-M allows an increase of $50 \%$ in terms of energy efficiency and $--\mathrm{dB}$ gain in terms of $E_{b} / N_{0}$ compared with classical SC-FDMA for the same spectral efficiency performance. In [12], index modulation has been applied to single carrier frequency domain equalization (SC-FDE) system, but the multiple access analysis as well as PAPR and BER performance evaluation for different access modes have not been studied in the previous work. In addition, our study is particularly applied to M2M and IoT applications.

Section II defines the principle of SC-FDMA-IM scheme, while Section III analyses the performance of the proposed scheme in terms of spectral efficiency, energy efficiency, PAPR, and BER for different access modes. Section IV concludes the paper and gives some directives for future works.

\section{SC-FDMA WITH INDEX MODULATION}

\section{A. SC-FDMA-IM principle}

Figure 1 depicts the principle of SC-FDMA-IM transceiver. Let $U$ be the total number of users. User $u$ has $m_{u}$ bits to transmit which are introduced to the index modulation block. IM processing for a user $u$ is based on the following parameters: $G_{u}$ is the number of groups within an SCFDMA-IM symbol of duration $T, n_{u}$ is the number of subcarriers in each group, and $k_{u}$ is the number of active subcarriers in each group. In our case, $k_{u}$ is supposed to be fixed for every group and during the transmission in order to reduce the complexity. A more flexible selection of the active number of sub-carriers has been proposed in [7] to increase the performance in terms of spectral efficiency.

A stream of $m_{u}=G\left(m_{1}^{u}+m_{2}^{u}\right)$ input information bits is mapped to a SC-FDMA-IM symbol associated to a 


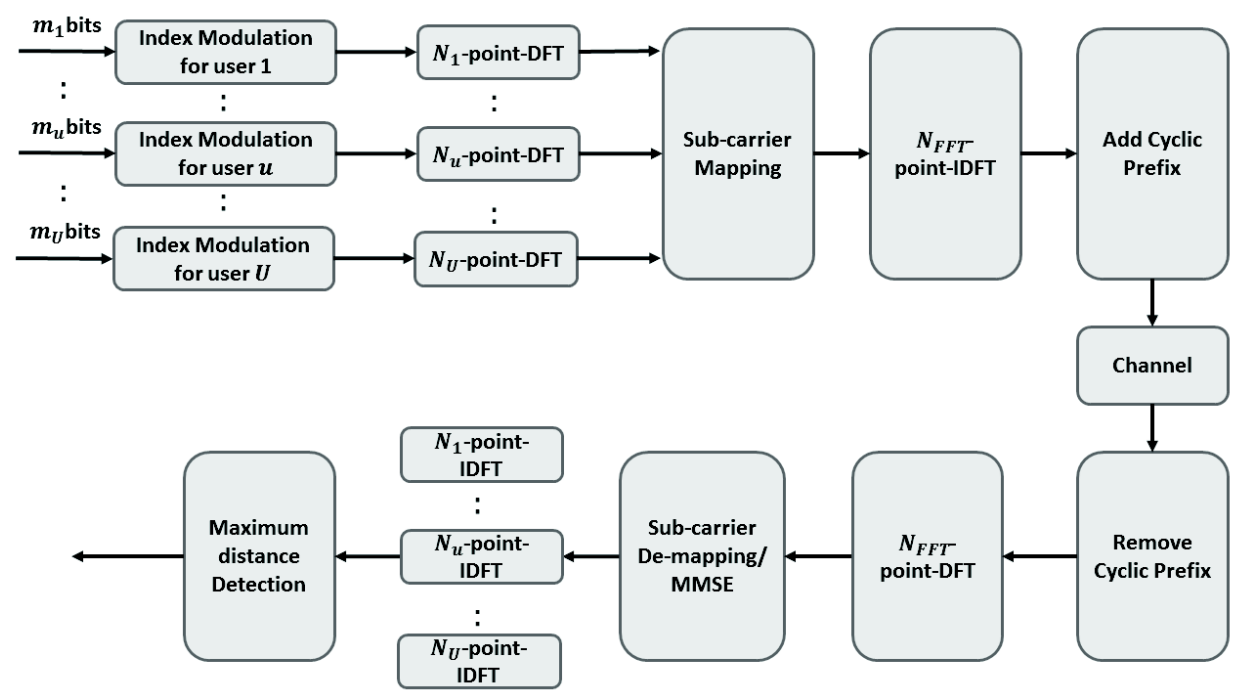

Figure 1: SC-FDMA-IM transceiver.

user $u$, where $m_{1}^{u}=\left|\log _{2}\left(\begin{array}{l}n_{u} \\ k_{u}\end{array}\right)\right|$ is the number of bits to be mapped to an index set of actives sub-carriers, and $m_{2}^{u}=k_{u} \log _{2}(M)$ is the number of bits mapped to $\mathcal{M}$-ary constellation symbols. The total number of active sub-carriers is denoted $K_{u}=k_{u} G_{u}$ and the total number of the allocated sub-carriers for a user $u$ is $N_{u}=n_{u} G_{u}$. The index selection is performed by applying a selection procedure such as a look-up table or a combinatorial mapping. More details on IM can be found in [4].

Dividing the input information bits $m_{u}$ into $G_{u}$ groups is relevant for the order of the implementation complexity. For $G_{u}=1$, the binomial coefficient $\left(\begin{array}{l}N_{u} \\ K_{u}\end{array}\right)$ can take large values which make the implementation of the system computationally unaffordable.

The index modulation procedure is applied for each user $u$, and then an $N_{u}$-point-DFT (discrete Fourier transform) is performed on the output vector, which contains symbols from a pre-defined constellation and zeros associated to the inactive sub-carriers. The DFT operation is followed by a sub-carrier mapping in order to establish the access mode of the users, and a $N_{\text {FFT-point-IDFT (inverse discrete Fourier }}$ transform) is then applied for each user separately. A similar inverse procedure is performed at the receiver side along with a channel equalization and a minimum distance detection.

\section{B. Multiple access modes}

Sub-carrier mapping mentioned in Figure 1 chooses the access mode for different users $u \in \llbracket 1, U \rrbracket$. In this work, we investigate the interleaved sub-carrier mapping and the localized sub-carrier mapping. In the interleaved sub-carrier access mode, the DFT outputs of the input data are allocated in equidistant frequency spacings over the entire bandwidth with zeros occupying the unused sub-carriers, whereas consecutive sub-carriers are occupied by the DFT outputs of the input data in the localized sub-carrier access mode. Note that there is difference between the aforementioned unused sub-carriers and the inactive sub-carriers related to index modulation. The unused sub-carriers in the access modes will be used for other users. The inactive sub-carriers resulting

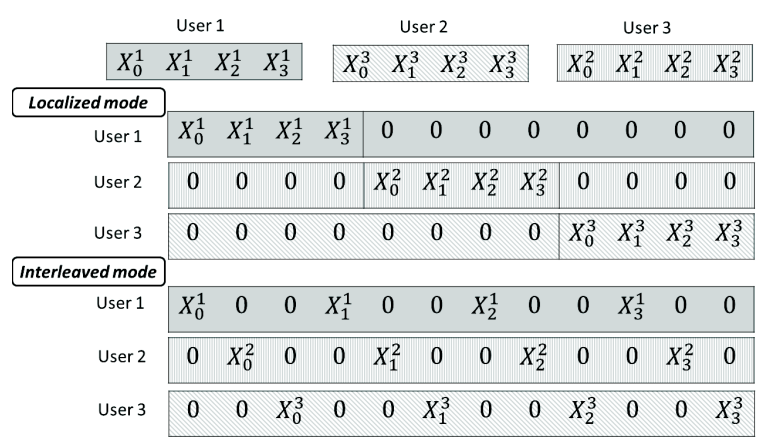

Figure 2: SC-FDMA-IM access modes. Example when number of users is equal to $U=3$ and $N_{\mathrm{FFT}}=8$

from index modulation block remain inactive during the whole transmission.

Let $\left\{X_{n}^{u}, 1 \leq u \leq U, 0 \leq n \leq N_{u}-1\right\}$ the data sequence at the output of the $N_{u}$ point DFT for user $u$. Figure 2 represents an example of the sub-carrier mapping modes for a number of users equal to $U=3, N_{\mathrm{FFT}}=8$, and $N_{u}=4$ for each user.

\section{Performance Analysis}

To demonstrate the interest of the proposed scheme for cellular IoT applications, SC-FDMA-IM and SC-FDMA performance are compared in terms of spectral efficiency, energy efficiency, PAPR, and BER for NB-IoT and LTE-M standards in both localized and interleaved modes.

\section{A. Spectral efficiency}

a) Spectral efficiency expression: Spectral efficiency is a relevant performance metric in communication systems. It relates to the data rate $B^{r}$ that can be achieved within a certain allocated bandwidth $\mathcal{W}$. It is formally defined as:

$$
\xi(\text { bits } / s / H z)=\frac{B^{r}(\text { bits } / s)}{W(H z)} .
$$

In SC-FDMA-IM, $k_{u}$ sub-carriers are selected out of $n_{u}$ for each group, and $\log _{2}(\mathcal{M})$ data bits are assigned to each 
Table I: Parameter settings for SC-FDMA-IM

\begin{tabular}{|c|c|c|c|}
\hline Parameters & Definition & $\begin{array}{c}\text { Value for } \\
\text { NB-IoT }\end{array}$ & $\begin{array}{c}\text { Value } \\
\text { for } \\
\text { LTE-M }\end{array}$ \\
\hline$N_{\mathrm{FFT}}$ & IFFT/FFT size & 72 & 256 \\
\hline$\Delta F$ & Inter-carrier spacing & $3.75 \mathrm{kHz}$ & $15 \mathrm{kHz}$ \\
\hline$T$ & $\begin{array}{c}\text { Duration of block of } \\
\text { symbols }\end{array}$ & $\frac{1}{\Delta F}$ \\
\hline$T_{\mathrm{cp}}$ & Duration of cyclic prefix & \multicolumn{2}{|c|}{$T / 8$} \\
\hline $\mathrm{RB}$ & Resource Block & 12 sub-carriers \\
\hline $\mathcal{M}$ & $\mathcal{M}$-QAM Constellation & $\begin{array}{c}4 \text { for SC-FDMA-IM } \\
\text { and 2 for SC-FDMA }\end{array}$ \\
\hline$r$ & $\begin{array}{c}\text { Roll-off of the root raised } \\
\text { cosine filter }\end{array}$ & \multicolumn{2}{|c|}{0.3} \\
\hline$L$ & Upsampling coefficient & \multicolumn{2}{|c|}{8} \\
\hline$\nu$ & $\begin{array}{c}\text { Number of path for } \\
\text { Rayleigh channel }\end{array}$ & $\begin{array}{c}10^{6} \text { for PAPR and } \\
\text { BER simulations }\end{array}$ \\
\hline$N_{\text {iter }}$ & Number of iterations \\
\hline
\end{tabular}

selected sub-carrier, the effective bit rate for each user $u$ is equal to:

$$
B_{u}^{r}=\frac{G_{u}}{T+T_{\mathrm{cp}}}\left(\left\lfloor\log _{2}\left(\begin{array}{c}
n_{u} \\
k_{u}
\end{array}\right)\right\rfloor+k_{u} \log _{2}(\mathcal{M})\right) .
$$

Knowing that the allocated bandwidth $W_{u}$ to a user $u$ is given by $\Delta F N_{u}$, we get the following spectral efficiency expression:

$$
\begin{aligned}
\xi_{\mathrm{IM}} & =\frac{\sum_{u=1}^{U} B_{u}^{r}}{\sum_{u=1}^{U} W_{u}} \\
& =\frac{\sum_{u=1}^{U} G_{u}\left(\left\lfloor\log _{2}\left(\begin{array}{c}
n_{u} \\
k_{u}
\end{array}\right)\right\rfloor+k_{u} \log _{2}(\mathcal{M})\right)}{\left(T+T_{\mathrm{cp}}\right) \Delta F \sum_{u=1}^{U} N_{u}}
\end{aligned}
$$

b) Spectral efficiency increase for different numbers of users: We define the spectral efficiency increase as

$$
\rho=\frac{\xi_{\mathrm{IM}}-\xi_{0}}{\xi_{0}},
$$

where $\xi_{0}$ is the spectral efficiency of the benchmark classical SC-FDMA.

In order to evaluate the spectral efficiency increase of NB-IoT applications, we consider the parameters shown in Table I. We assume that each user is allocated at least an integer number of resource blocks (RB), such that the length of each RB is 12 sub-carriers. Knowing that the allocated bandwidth counts $6 \mathrm{RB}$, the possible number $\mu$ of users that can have access to the allocated bandwidth at the same time is then $\mu \in \llbracket 1,6 \rrbracket$. Adding the assumption that the bandwidth $W$ is allocated equally to each user, the possible number of users becomes $\mu \in\{1,2,3,6\}$. In fact, we can have one user that occupies the whole bandwidth, or 2 users with $3 \mathrm{RB}$ each, or 3 users with $2 \mathrm{RB}$ each, or 6 users with $1 \mathrm{RB}$ each.

Hereafter, we consider that, for each transmission all the users divide their allocated bandwidth $W_{u}$ to the same number of groups. The values of the number of groups for each user depend then on the number of users $\mu$. For each group of sub-carriers, only round $\left(\alpha n_{u}\right)^{2}$ are active out of $n_{u}$, such that $\alpha$ is chosen to be equal to $\frac{1}{4}$ in this example. Other

${ }^{2}$ round(.) is a function that rounds the input to the nearest integer.

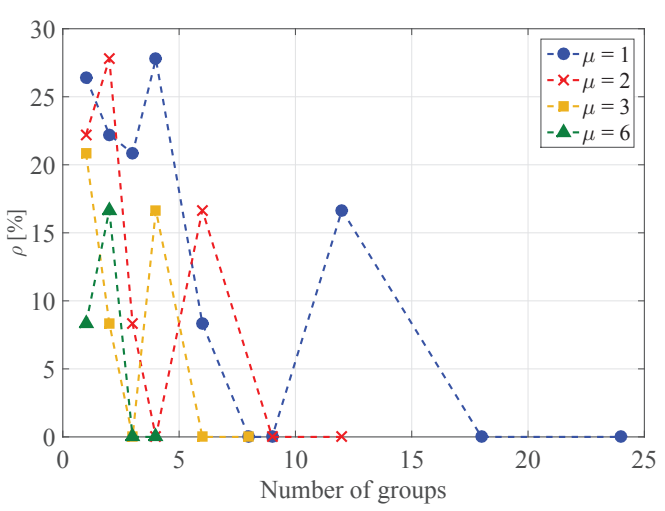

Figure 3: Spectral efficiency increase of different numbers of users for different numbers of groups.

values of $\alpha$ can be selected from the interval $[0,1]^{3}$. Eq. (5) becomes:

$$
\rho(\mu)=\frac{G^{\mu}\left(\left\lfloor\log _{2}\left(\begin{array}{c}
n^{\mu} \\
k^{\mu}
\end{array}\right)\right\rfloor+k^{\mu} \log _{2}(\mathcal{M})\right)}{\log _{2}(\sqrt{\mathcal{M}})}-1 .
$$

Eq. (6) is represented in Figure 3 for different numbers of groups when $\mu \in\{1,2,3,6\}$. We can observe that, in general, increasing the number of users reduces the spectral efficiency of SC-FDMA-IM. This can be explained by the fact that, for the same total allocated bandwidth, when we reduce the number of users, the index modulation of each user is applied to a larger bandwidth, which allows groups with larger lengths, the number of possible active sub-carriers combinations gets higher. Hence the increase in terms of spectral efficiency.

c) Suitable number of groups: The different curves in Figure 3 indicate that the spectral efficiency depends on the number of groups $G_{\mu}$ considered for each scenario $\mu$. Grouping the data bits reduces the number of possible combinations from $\left(\begin{array}{l}N_{u} \\ K_{u}\end{array}\right)$ to $G_{u}\left(\begin{array}{c}N_{u} / G_{u} \\ K_{u} / G_{u}\end{array}\right)$, which reduces the complexity of the detection at the receiver side, since the detection of the used combination of active sub-carriers is performed over each group. From Figure 3, we can observe that the following number of groups achieves the same spectral efficiency than classical SC-FDMA i.e the spectral efficiency increase is set to zero $(\rho=0)$, while having a small number of sub-carriers ( 3 or 4 ) and then reduced complexity:

$$
\left(G_{\mu}, n_{\mu}, k_{\mu}\right) \in\left\{\begin{array}{ll}
(\{18,24\},\{4,3\}, 1) & \text { if } \mu=1 \\
(\{9,12\},\{4,3\}, 1) & \text { if } \mu=2 \\
(\{6,8\},\{4,3\}, 1) & \text { if } \mu=3 \\
(\{3,4\},\{4,3\}, 1) & \text { if } \mu=6
\end{array} .\right.
$$

\section{B. Energy efficiency}

In several IoT and M2M applications (especially massive M2M schemes), the spectral efficiency may not be the only most relevant performance criteria. Numerous applications tolerate a spectral efficiency decrease in order to save energy so that the batteries lifetime lasts longer. In order to

\footnotetext{
${ }^{3}$ In [11], the authors have proven using Stirling's approximation of the binomial coefficient, that for a large number of sub-carriers, $\alpha$ approaches a spectral efficiency maximizing value given by $\alpha \underset{N \rightarrow \infty}{=} \frac{\mathcal{M}}{\mathcal{M}+1}$.
} 
save energy in SC-FDMA-IM, we will activate the lowest possible number of active sub-carriers while maintaining the same spectral efficiency than classical SC-FDMA and a low implementation complexity. The following configuration is then chosen:

$$
\left(G_{\mu}, n_{\mu}, k_{\mu}\right) \in\left\{\begin{array}{ll}
(18,4,1) & \text { if } \mu=1 \\
(9,4,1) & \text { if } \mu=2 \\
(6,4,1) & \text { if } \mu=3 \\
(3,4,1) & \text { if } \mu=6
\end{array} .\right.
$$

We define the energy efficiency $\mathcal{E}$ (bits/Joule) as the total amount of bits normalized by the energy. The energy efficiency increase is then expressed as

$$
\mathcal{R}=\frac{\mathcal{E}_{\mathrm{IM}}-\mathcal{E}_{0}}{\mathcal{E}_{0}}
$$

where the index IM relates to SC-FDMA-IM, and the index 0 stands for the benchmark SC-FDMA. Applying the settings of Eq. (8), the energy efficiency increase is equal to:

$$
\begin{aligned}
\mathcal{R} & =\frac{G_{\mu} k_{\mu} \log _{2}(\mathcal{M})-G_{\mu} n_{\mu} \log _{2}(\sqrt{\mathcal{M}})}{G_{\mu} n_{\mu} \log _{2}(\sqrt{\mathcal{M}})} \\
& =-50 \% .
\end{aligned}
$$

Thanks to its ability to carry more data while sending less number of bits, the introduction of index modulation reduces the energy consumption by $50 \%$ which is a significant improvement, especially for low-power wide-area networks.

Similar analysis of spectral efficiency and energy efficiency can be conducted for LTE-M using the parameters in Table I. It leads to the same significant result of $50 \%$ energy consumption reduction following the same aforementioned steps.

\section{PAPR performance}

In this section, the PAPR performance is studied using the parameter settings reported in Table I. For NB-IoT, we assume that 6 users shares equally the bandwidth $\mu=6$ (1 resource block for each user). In LTE-M, the user occupies 6 resource blocks. For each user, the grouping is performed so that the total number of sub-carriers per group $n$ and the active number of sub-carriers $k$ within this group are set to $(n, k)=(4,1)$. These settings insure reaching $50 \%$ of energy efficiency increase and a same spectral efficiency as SC-FDMA along with low implementation complexity as given by Eq. (8) and Eq. (11). In fact, since only one subcarrier is activated in a group of length 4 sub-carriers, the number of combinations per group is reduced to 4 .

The PAPR performance of NB-IoT and LTE-M for localized and interleaved access modes is evaluated through the complementary cumulative distribution function (CCDF) which is the probability that the PAPR exceeds some value $\gamma$. The results represented in Figure 4 and Figure 5 show that the introduction of index modulation in SC-FDMA for NB-IoT and LTE deteriorates the PAPR performance for the difference access modes. The resulting difference in terms of PAPR performance becomes less important when we increase the number of sub-carriers (the length of the IDFT). Hence, it is important to apply a PAPR reduction technique to address this issue.
Table II: Look-up table.

\begin{tabular}{|c|c|}
\hline Incoming bits $m_{1}$ & Associated index set \\
\hline 00 & 1 \\
\hline 10 & 2 \\
\hline 10 & 3 \\
\hline 11 & 4 \\
\hline
\end{tabular}

\section{BER performance}

The BER performance is evaluated in a frequency-selective Rayleigh fading channel represented by the channel impulse response coefficients: $h=(h(1), h(2) \ldots h(\nu))$. We assume that all the coefficients are circularly symmetric complex Gaussian random variables with $\mathcal{C N}\left(0, \frac{1}{\nu}\right)$ distribution. The associated BER performance results are displayed in Figure 6 for NB-IoT and in Figure 7 for LTE-M. For signal recovery, a minimum mean square error (MMSE) equalization is employed along with a minimum distance detector. In order to provide a mapping between the index set of active subcarriers within a group and incoming bits, a mapping table is implemented in both transmitter and receiver sides. The lookup table selection is given in Table II. The same simulation parameters employed in Section III-C are considered in this part.

We can see that the SC-FDMA-IM scheme achieves a gain of $--\mathrm{dB}\left(--\mathrm{dB}\right.$ respectively) in terms of $E_{b} / N_{0}$ for $\mathrm{BER}=10^{-4}$ for NB-IoT (LTE-M respectively). The index modulation allows more favourable spectrum spacing than the classical modulation, which explains the interesting improvement in BER performance.

\section{CONCLUSION}

In this paper, we have introduced the index modulation to the SC-FDMA scheme for uplink M2M and IoT transmissions using LTE-M and NB-IoT standards for both localized and interleaved access modes for multiple users. For the same spectral efficiency as classical SC-FDMA-IM, we have showed that index modulation can lead to a gain of $50 \%$ in terms of energy efficiency while maintaining an affordable transceiver complexity. Due to favourable spectrum spacing resulting from index modulation, the BER is improved by up to $--\mathrm{dB}$ at the cost of an acceptable PAPR increase. We believe that this significant result encourages more studies about SC-FDMA-IM, especially for IoT and M2M applications.

\section{ACKNOWLEDGMENT}

This work has received a French state support granted to the Enhanced PHY for cellular Low power communication IoT (EPHYL) project and managed by the National Research Agency under reference Nb. ANR-16-CE25-0002-03.

\section{REFERENCES}

[1] Usman Raza, Parag Kulkarni, and Mahesh Sooriyabandara. Low Power Wide Area Networks: An Overview. IEEE Communications Surveys \& Tutorials, 2017.

[2] Hamidreza Shariatmadari, Rapeepat Ratasuk, Sassan Iraji, Andrés Laya, Tarik Taleb, Riku Jäntti, and Amitava Ghosh. Machine-type communications: current status and future perspectives toward $5 \mathrm{G}$ systems. IEEE Communications Magazine, 53(9):10-17, 2015. 


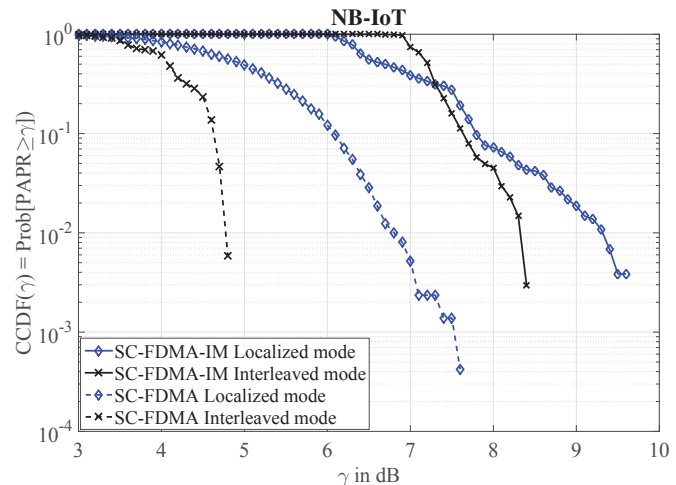

Figure 4: PAPR performance for SC-FDMA-IM in NBIoT scenario.

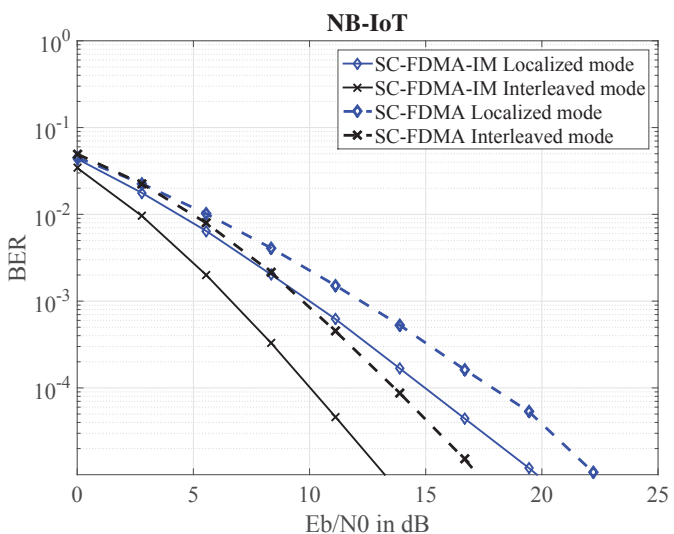

Figure 6: BER performance for SC-FDMA-IM in NBIoT scenario.

[3] Nitin Mangalvedhe, Rapeepat Ratasuk, and Amitava Ghosh. NB-IoT deployment study for low power wide area cellular IoT. In Personal, Indoor, and Mobile Radio Communications (PIMRC), 2016 IEEE 27th Annual International Symposium on, pages 1-6. IEEE, 2016.

[4] Ertuğrul Başar, Ümit Aygölü, Erdal Panayırc1, and H Vincent Poor. Orthogonal Frequency Division Multiplexing with Index Modulation. IEEE Transactions on Signal Processing, 61(22):5536-5549, 2013.

[5] Pal K Frenger and N Arne B Svensson. Parallel combinatory OFDM signaling. IEEE transactions on communications, 47(4):558-567, 1999.

[6] Roberto Padovani and J Wolf. Coded Phase/Frequency Modulation. IEEE transactions on communications, 34(5):446-453, 1986.

[7] Rui Fan, Ya Jun Yu, and Yong Liang Guan. Generalization of Orthogonal Frequency Division Multiplexing with Index Modulation. IEEE Transactions on Wireless Communications, 14(10):5350-5359, 2015

[8] Yue Xiao, Shunshun Wang, Lilin Dan, Xia Lei, Ping Yang, and Wei Xiang. OFDM with interleaved subcarrier-index modulation. IEEE Communications Letters, 18(8):1447-1450, 2014.

[9] E. Başar. Index modulation techniques for $5 \mathrm{G}$ wireless networks. 54(7):168-175, 2016.

[10] Sungnam Hong, Min Sagong, Chiwoo Lim, Kyungwhoon Cheun, and Sunghye Cho. FQAM: A Modulation Scheme for Beyond 4G Cellular Wireless Communication Systems. In 2013 IEEE Globecom Workshops (GC Wkshps), pages 25-30. IEEE, 2013.

[11] Marwa Chafii, Justin P Coon, and Dene A Hedges. DCT-OFDM with Index Modulation. IEEE Communications Letters, 2017.

[12] Miyu Nakao, Takumi Ishihara, and Shinya Sugiura. Single-carrier frequency-domain equalization with index modulation. IEEE Communications Letters, 21(2):298-301, 2017.

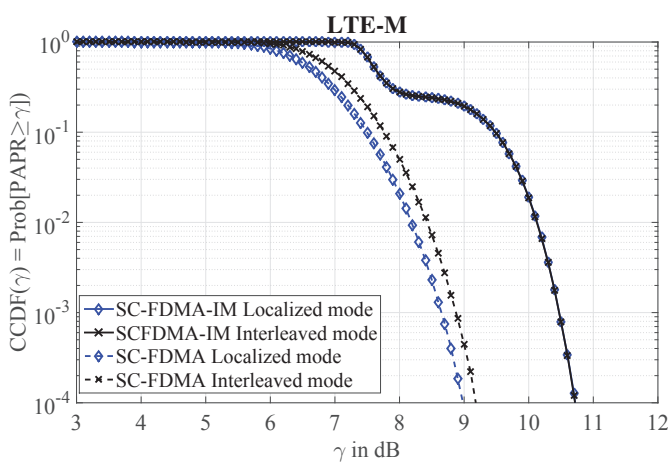

Figure 5: PAPR performance for SC-FDMA-IM in LTEM scenario.

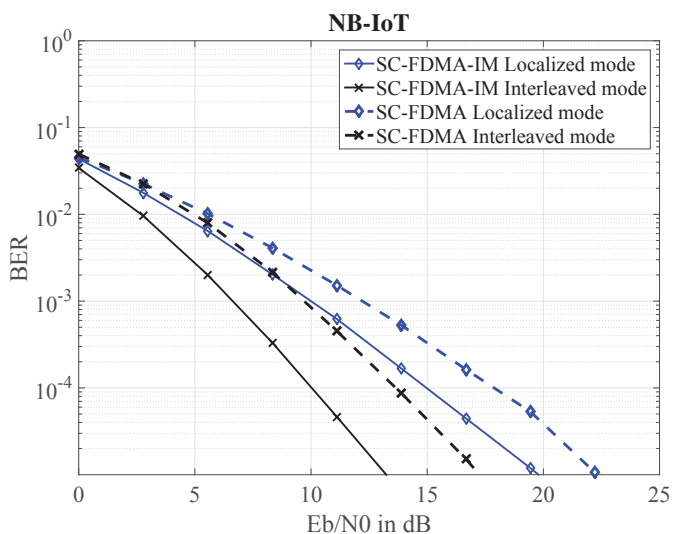

Figure 7: BER performance for SC-FDMA-IM in LTEM scenario. 